\title{
Repulsion of phase-velocity dispersion curves and the nature of plate vibrations
}

\author{
M. DESCHAMPS, B. HOSTEN, A. GÉRARD and H. ÜBERALL*
}

\author{
Laboratoire de Mécanique Physique, Université Bordeaux I, 33405 Talence, France \\ * Department of Physics, Catholic University of America, Washington, DC 20064, U.S.A.
}

\begin{abstract}
The Lamb waves propagating in an elastic plate in vacuo generate compressional $(\mathrm{L})$ and shear type (T) plate vibrations which are coupled due to the boundary conditions. Without such coupling, their phase-velocity dispersion curves would form two intersecting families which at high frequency tend towards the elastic-wave speeds $\mathrm{C}_{\mathrm{L}}$ and $\mathrm{C}_{\mathrm{T}}$, respectively. It is shown that the coupling causes a repulsion of the dispersion curves similar to that encountered in atomic physics for the energy levels of atoms combining into molecules, which prevents their intersection and at the same time exchanges the nature $(\mathrm{L} \leftrightarrow \mathrm{T}$ ) of the underlying vibrations. However, in the repulsion regions a succession of dispersion curves combines to asymptotically approach the uncoupled $\mathrm{L}$ or $\mathrm{T}$ dispersion curves, respectively.
\end{abstract}

\section{1-INTRODUCTION}

One refers to the propagating modes of vibration of an elastic plate in vacuo as Lamb waves 1. Due to the symmetry of the problem about the median plane of the plate, there exists a family of symmetric Lamb modes denoted $\mathrm{S}_{0}, \mathrm{~S}_{1}, \mathrm{~S}_{2} \ldots$, and an antisymmetric family $\mathrm{A}_{0}, \mathrm{~A}_{1}, \mathrm{~A}_{2} \ldots$, with well-known dispersion curves shown, e.g., for an aluminum plate in Fig. 1. The material parameters of $\mathrm{Al}$ were taken here as the longitudinal bulk velocity $\mathrm{C}_{\mathrm{L}}=6.400 \mathrm{~mm} / \mu \mathrm{s}$, shear velocity $\mathrm{C}_{\mathrm{T}}=3.100 \mathrm{~mm} / \mu \mathrm{s}$, and mass density $\rho=2,780 \mathrm{~kg} / \mathrm{m}^{3}$. With the exception of the $A_{0}$ and $S_{0}$ waves which exist down to arbitrarily low frequencies, all $A_{i}$ and $S_{i}$ propagating modes exist only above individual cutoff frequencies at the points where their phase velocities tend to infinity. In the limit of infinite frequency, the $A_{0}$ and $S_{0}$ curves tends towards the Rayleigh wave velocity $C_{R}$ on a half-space $\left({ }^{1}\right)$, while all other curves, after having lingered for a while around the value of $\mathrm{C}_{\mathrm{L}}$, finally descend and approach the value of $\mathrm{CT}_{\mathrm{T}}$. There are, incidentally, some $S_{i}$ curves that intersect $A_{i}$ curves; this phenomenon was studied recently by $Z$ hu and Mayer $\left({ }^{2}\right)$ who showed that around the crossover points, Lamb modes do not exist. We shall here be interested in the physical nature of the Lamb waves, and we ask whether a predominantly longitudinal or shear (flexural) character of a given Lamb mode can be established from the dispersion curves alone, without having to proceed to a calculation of displacements as was done in Ref. (1).

\section{THE PLATE IN VACUO : PERTURBATION BY THE COUPLING OF ELASTIC WAVES}

The characteristic equation for the vibrations of a plate in vacuo, which can be solved for the dispersion curves of the phase velocities $C_{i}$ of the Lamb modes $A_{i}$ and $S_{i}$, is given in textbooks such as Refs. $\left({ }^{1}\right)$ or $\left(^{9}\right)$. For the more general case of two different fluids with sound speeds $C_{j}$ and densities $\rho_{j}(j=$ $1,2)$ bounding the plate on opposite sides and using a notation similar to that of Talmant, Ref. $(5)$, it is given by : 


$$
\begin{gathered}
\sum_{j=1}^{2} S_{j}^{f} A_{j}^{f}-\frac{1}{\rho^{2}}\left(\frac{\rho_{1}}{R_{1}}-\frac{\rho_{2}}{R_{2}}\right)^{2} k_{\Gamma}^{8} R_{L}^{2}=0, \\
S_{j}^{f}=4 k^{2} R_{T} R_{L} \tanh \left(R_{L} d / 2\right)-\left(R_{T}^{2}+k^{2}\right)^{2} \tanh \left(R_{T} d / 2\right) \\
-k_{T}^{4}\left(\rho_{j} R_{L} / \rho R_{j}\right) \tanh \left(R_{L} d / 2\right) \tanh \left(R_{T} d / 2\right),
\end{gathered}
$$

and $A_{j}^{f}$ originates from $S_{j}^{f}$ by replacing every tanh by coth. For the case considered in the present section, i.e. $\rho_{j}=0$ (plate in vacuo) we introduce the notation $S$ and $A$ for the corresponding quantities. Further, $R_{m}=2 \pi f\left(C_{i}^{-2}-C_{m}^{-2}\right)^{1 / 2}$ where $m=T, L, 1$ or 2 , and $C_{i}$ in the phase velocity of the $i$ th plate mode, to be determined ; and $\mathrm{kT}_{\mathrm{T}}=\omega / \mathrm{C}_{\mathrm{T}}, \omega=2 \pi \mathrm{f}$, while $\mathrm{k}$ is the propagation constant in the direction parallel to the plate. Solving Eq (1) with $\rho_{j}=0$ furnishes the curves of Fig. 1. In order to understand their features, which are marked by the coupling of compressional and shear waves through the boundary conditions of vanishing stresses at the interfaces, we may consider separately the limiting cases of (a) a fluid layer in vacuo $\left(C_{T}=0\right)$, and $(b)$ an artificial layer in vacuo which only supports shear waves $\left(C_{L}=0\right)$. The dispersion curves of the corresponding first few families of plate modes are shown in Fig. 2, marked by $\mathrm{C}_{\mathrm{T}}=0$ or $\mathrm{C}_{\mathrm{L}}=0$, respectively (i.e. being correspondively of purely compressional of purely shear character).

These two intersecting families of curves asymptotically approach the values of $\mathrm{C}_{\mathrm{L}}$ or $\mathrm{C}_{\mathrm{T}}$, respectively, in the limit of $\mathrm{fd} \rightarrow \infty$, which reveals their physical character as that of compressional or of shear waves, respectively. Although presented together in Fig. 2, these two families are not simultaneously present since by e.g. setting $\mathrm{C}_{\mathrm{T}}=0$, we not only remove the coupling between $\mathrm{L}$ and $\mathrm{T}$ waves, but we remove the $\mathrm{T}$ waves altogether. In order to verify more directly the character of the dispersion curves, we carry out a perturbation treatment of Eq. (1) (with $\rho_{j}=0$ ), starting out with the fluid layer $\left(C_{T}=0\right)$ taken as the unperturbed case for which the phase velocity is designated as $\mathrm{C}_{j}^{(0)}$. This corresponds, of course, to the dispersion curve family denoted by $\mathrm{C}_{\mathrm{T}}=0$ in Fig.2, i.e., having purely compressional character. Subsequently, we insert :

$$
\mathrm{C}_{\mathrm{j}}=\mathrm{C}_{\mathrm{j}}^{(0)}\left(1+\delta_{0}\right)
$$

in Eq. (1) (with $\rho_{j}=0$ ), where the correction is assured of first order in $C_{T}$, and carry out a Taylor-series expansion in $\mathrm{C}_{\mathrm{T}}$ of Eq. (1), with details given in Ref. (10). This calculation shows that the correction to the Lamb speed in a fluid layer contains the dispersion equation of shear waves, and vice-versa.

The resulting values for $C_{j}$ are shown in Fig. 3 plotted as solid curves. It is immediately apparent here that these "quasi-fluid", or "quasi-compressional" dispersion curves reproduce closely the near-flat segments of the exact curves, revealing their compressional character. It is also seen that the steeply deviating portions of the exact as compared to the fluid-layer curves (following their repulsions), being initially reproduced by our first-order (in $\mathrm{C}_{\mathrm{T}}$ ) perturbation results, solid curves, are in fact due to the effect of the perturbation caused by the interaction of compressional and shear vibrations ; this reveals the increasing shear character of the steep portions of the dispersion curves, just as well as their eventual convergence at the value $\mathrm{C}_{\mathrm{T}}$ for $\mathrm{fd} \rightarrow \infty$, where they have turned $100 \%$ transverse ${ }^{1}$ ). The corresponding change of physical character of the dispersion curves, after having passed through an episode of repulsion, corresponds exactly to the same phenomenon observed in the atomic case $(3)$.

For the case of a plate bounded by fluid on one side, and vacuum on the other, the dispersion curves of the fluid-borne (Stoneley-Scholte type) wave which is known from the studies of Grabowska $\left({ }^{4}\right)$ and Talmant $\left({ }^{1}\right)$ to be present. In such a case, a fluid-borne wave referred to as "A-wave" $(5,6)$, and often identified with the Scholte-Stoneley wave observed in related circumstances $(7,8)$, is present which exhibits, jointly with the $A_{0}$ Lamb wave, a similar repulsion phenomenon. This latter is caused by the presence of the fluid as a perturbation $\left({ }^{10}\right)$. Analogs of $A_{0}$ and $A$ waves, quite similar to those of the Grabowska plate case discussed in this paper, have also been found by calculations of circumferentialwave dispersion curves on spherical $(11,6)$ and cylindrical $(12,13)$ water-loaded, air-filled shells. 


\section{CONCLUSIONS}

On the basis of perturbation considerations, we have clarified the physical character ( $\mathrm{L}$ or $\mathrm{T}$ ) of the Lamb waves on elastic plates in vacuo along separate portions of their dispersion curves. The coupling between $\mathrm{L}$ and $\mathrm{T}$ vibrations of the plate, which is caused by the boundary conditions, generates successive changes in character of a given Lamb wave along its dispersion curve, and also causes repulsions between neighboring dispersion curves of a given symmetry (symmetric or antisymmetric) that prevent then from

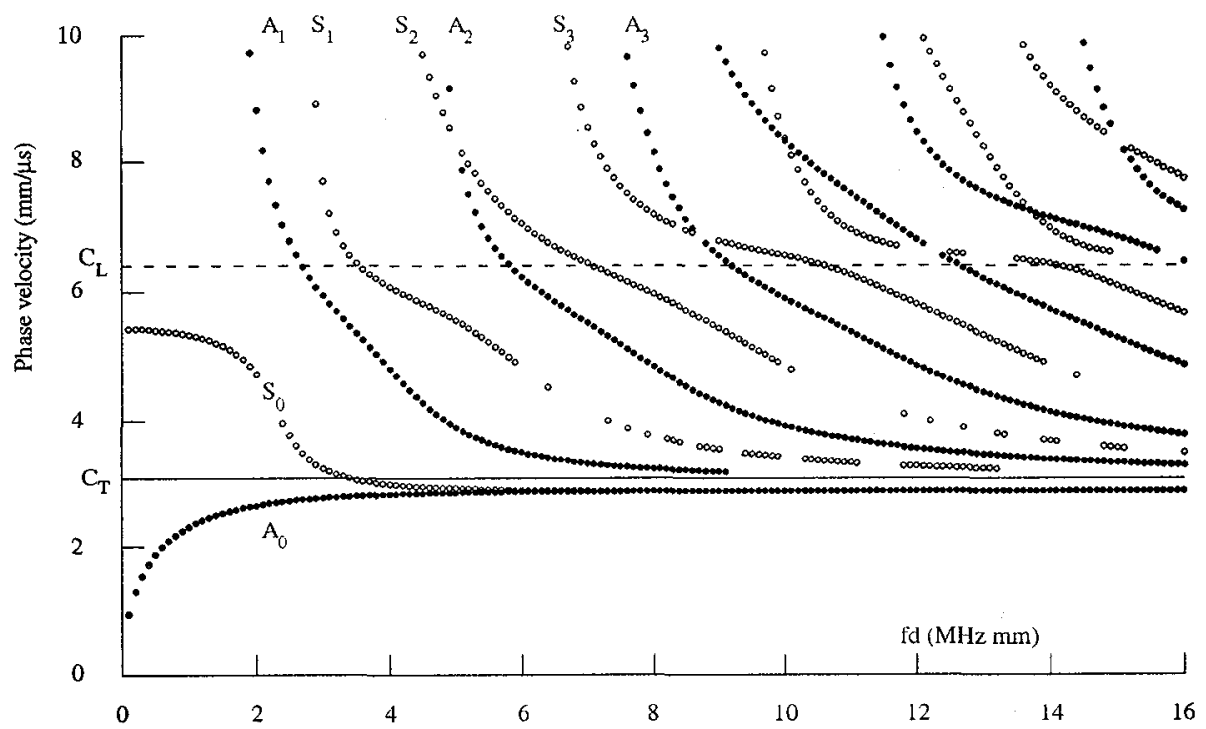

Fig. 1: Dispersion curves of phase velocity versus the frequency-thickness product fd.

- : antisymmetric modes ; 0 : symmetric modes. - - : : $\mathrm{CL}_{\mathrm{L}}=6.4 \mathrm{~mm} / \mu \mathrm{s} ; \_$: $\mathrm{C}_{\mathrm{T}}=3.1 \mathrm{~mm} / \mu \mathrm{s}$.

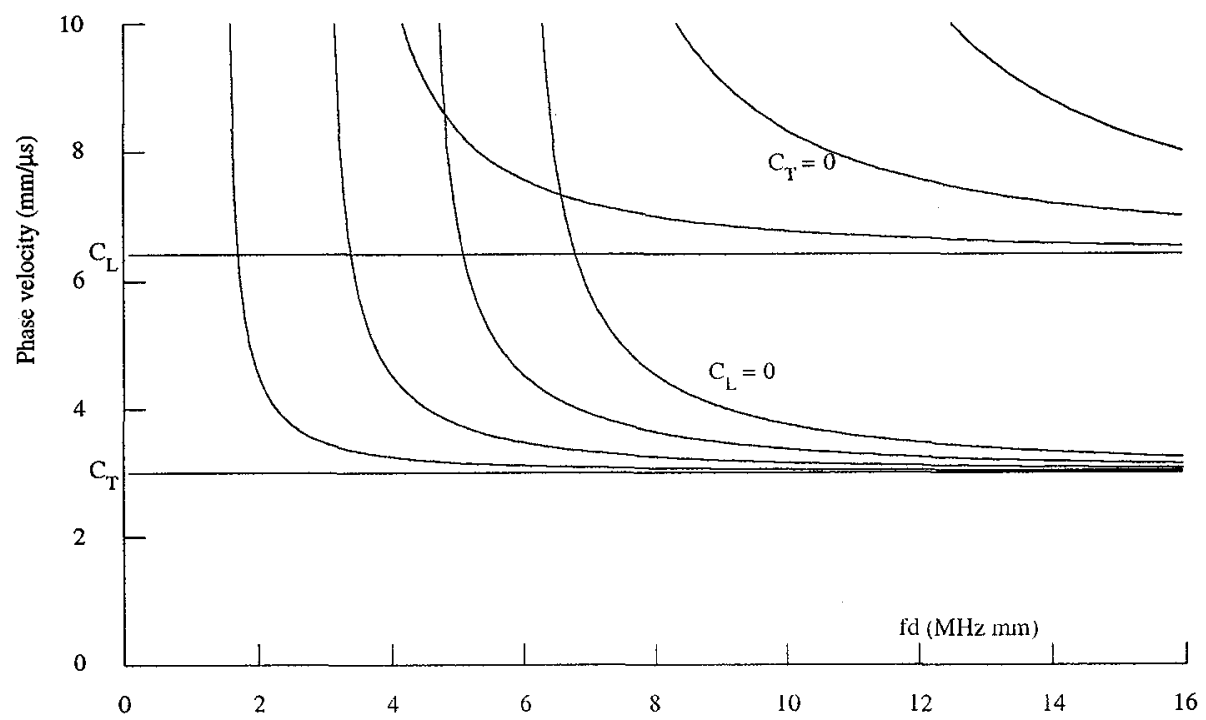

Fig. 2: Dispersion curves of a fluid plate in vacuo with sound speed $\mathrm{C}_{\mathrm{L}}$ equal to that in aluminum (marked $\mathrm{C}_{\mathrm{T}}=0$ ), and of an artificial plate supporting shear only, in vacuo, with shear speed $\mathrm{C}_{\mathrm{T}}$ equal to that in aluminum (marked $\mathrm{CL}_{\mathrm{L}}=0$ ). 


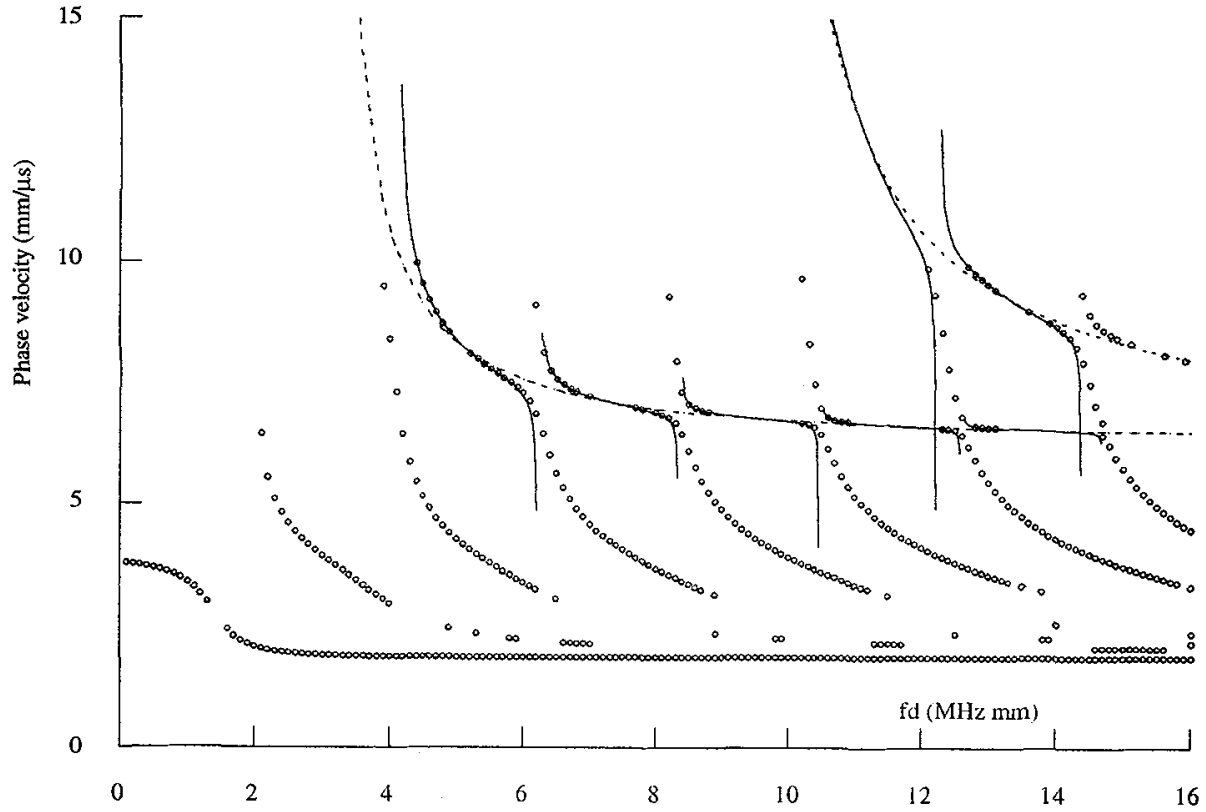

Fig.3 : Symmetric dispersion curve, and their approximation by first-order perturbation theory in $\mathrm{C}_{\mathrm{T}}$, starting from the purely compressional case (solid curves).

intersecting. For decreasing perturbation $\left(\mathrm{C}_{\mathrm{T}} \rightarrow 0\right.$ or $\left.\mathrm{C}_{\mathrm{L}} \rightarrow 0\right)$, segments of successive dispersion curves line up to approximate the uncoupled dispersion curves of purely $\mathrm{L}$ type (fluid layer) or of purely $\mathrm{T}$ type (artificial layer that supports shear only), respectively. The character of a Lamb wave switches as the dispersion curve passes through a repulsion region, but the original character is carried over to the corresponding segment of the adjacent dispersion curve.

\section{REFERENCES}

[1]. I.A. Viktorov, Rayleigh and Lamb Waves: Physical Theory and Applications (Plenum, New York, 1967).

[2]. Q. Zhu and G.W. Mayer,, J. Acoust. Soc. Am. 93, 1983-1895 (1993).

[3]. See e.g., P.H.E. Meijer and E. Bauer, Group Theory, North Holland, Amsterdam, 1965, p.52.

[4]. A. Grabowska, Arch. Acoust. 4, 57-64 (1979).

[5]. M. Talmant, "Rétrodiffusion d'une impulsion ultrasonore brève par une coque cylindrique a paroi mince", Ph D Thesis, University of Paris VII, 1987.

[6]. M. Talmant, H. Überall, R.D. Miller, M.F. Werby and J.W. Dickey, . J. Acoust. Soc. Am. 86, 278-289 (1989).

[7]. R. Stoneley,, Proc. Roy. Soc. London A106, 416-428 (1924).

[8]. J.C. Scholte, "On the Stoneley wave equation", Proc. Kon. Ned. Akad. Van Wetensch., Amsterdam, 45, part I, pp.20-25, part II, pp.159-164 (1942).

[9]. L.M. Brekhovsikh, Waves in Layered Media, Academic Press, New York, 1960.

[10]. H. Überall,B. Hosten, M. Deschamps and A. Gérard, submitted to J. Acoust. Soc. Am.

[11]. G.S. Sammelmann, D.H. Trivett and R.H.Hackman,. J. Acoust Soc. Am. 85, 114-124 (1989).

[12]. G. Maze, F. Léon, J. Ripoche and H. Überall, submitted to Acustica.

[13]. G. Maze, F. Léon, J. Ripoche and H. Überall, submitted to J. Acoust. Soc. Am. 